\title{
Endoscopic ultrasound-guided pancreaticobiliary intervention in patients with surgically altered anatomy and inaccessible papillae: A review of current literature
}

\author{
Aaron Martin, Charles Andrew Kistler, Piotr Wrobel, Juliana F. Yang ${ }^{1}$, Ali A. Siddiqui \\ Division of Gastroenterology and Hepatology, Thomas Jefferson University, Philadelphia, PA, ${ }^{1}$ Division of Gastroenterology \\ and Hepatology, University of Texas Southwestern Medical Center, Dallas, TX, USA
}

\begin{abstract}
The management of pancreaticobiliary disease in patients with surgically altered anatomy is a growing problem for gastroenterologists today. Over the years, endoscopic ultrasound (EUS) has emerged as an important diagnostic and therapeutic modality in the treatment of pancreaticobiliary disease. Patient anatomy has become increasingly complex due to advances in surgical resection of pancreaticobiliary disease and EUS has emerged as the therapy of choice when endoscopic retrograde cholangiopancreatography failed cannulation or when the papilla is inaccessible such as in gastric obstruction or duodenal obstruction. The current article gives a comprehensive review of the current literature for EUS-guided intervention of the pancreaticobiliary tract in patients with altered surgical anatomy.
\end{abstract}

Key words: Biliary tract, endosonography, major pancreatic duct, surgically altered anatomy

\section{INTRODUCTION}

The management of pancreaticobiliary disease in patients with surgically altered anatomy (SAA) is a growing problem for gastroenterologists today. This is in part due to the increasing number of patients undergoing bariatric surgery for obesity, as well as the increasing number of patients having surgical resections and palliative procedures for pancreatic, biliary, and gastric cancers. Common surgical interventions performed for these diseases include Billroth II, Whipple operation, and Roux-en-Y reconstructions

\begin{tabular}{|l|l|}
\hline \multicolumn{2}{|c|}{ Access this article online } \\
\hline Quick Response Code: & Website: \\
\hline & www.eusjournal.com \\
\cline { 2 - 2 } & DOI: \\
\hline
\end{tabular}

either with intact papillae or bilioenteric anastomoses. ${ }^{[1,2]}$ As the number of patients undergoing these surgeries continues to increase, gastroenterologists will inevitably be required to manage a variety of pancreaticobiliary diseases in patients with SAA.

Endoscopic retrograde cholangiopancreatography (ERCP) is an essential tool used in the treatment and diagnosis of pancreaticobiliary disease. ERCP is

\footnotetext{
This is an open access article distributed under the terms of the Creative Commons Attribution-NonCommercial-ShareAlike 3.0 License, which allows others to remix, tweak, and build upon the work non-commercially, as long as the author is credited and the new creations are licensed under the identical terms.
}

For reprints contact: reprints@medknow.com

How to cite this article: Martin A, Kistler CA, Wrobel P, Yang JF, SiddiquiAA. Endoscopic ultrasound-guided pancreaticobiliary intervention in patients with surgically altered anatomy and inaccessible papillae: A review of current literature. Endosc Ultrasound 2016;5:149-56.

\section{Address for correspondence}

Dr. Ali A. Siddiqui, Division of Digestive and Liver Diseases, Thomas Jefferson University, Philadelphia, PA 19107, USA.

E-mail: aas138@jefferson.edu

Received: 2015-08-11; Accepted: 2016-03-05 


\section{Martin, et al.: Therapeutic EUS in altered anatomy}

challenging in patients with SAA; the prior interventions frequently result in long afferent limbs of small bowel making it difficult to access the papilla. Some of the common challenges encountered with ERCP in patients with SAA include excessive looping of the endoscope, difficulty in accessing the papilla, stenotic anastomotic sites, and a lack of provider experience in advanced endoscopic techniques. ${ }^{[3,4]}$ The overall success rate of ERCP using conventional equipment in patients with SAA is approximately $51 \%$, with a complication rate as high as $20 \%{ }^{[5,6]}$

Given the low success rate and increased complications of ERCP in patients with SAA, endoscopists have turned to percutaneous, laparoscopic, and advanced endoscopic options. Laparoscopic and percutaneous options are invasive, require coordination of multiple treatment teams, and have high complication rates reported at $13 \% \%^{[7]}$ and $25 \%$, respectively. ${ }^{[8,9]}$ Overtubeassisted enteroscopy (OAE) has improved the success rate of ERCP in patients with SAA; a recent review examining its use in patients with SAA demonstrated an overall success rate of $74 \% \cdot{ }^{[3]}$ However, given the variable success rates of OAE-assisted ERCP and the limited amount of gastroenterologists trained in OAE, providers continue to search for other options.

Endoscopic ultrasound (EUS) has emerged as an important diagnostic and therapeutic modality in the treatment of pancreaticobiliary disease. The use of EUS to safely gain access to the pancreaticobiliary system in patients with SAA is growing. As surgically altered patient anatomy has become increasingly complex, EUS has emerged as the therapy of choice when ERCP fails cannulation or when the papilla is inaccessible.

\section{ENDOSCOPIC ULTRASOUND-GUIDED BILIARY DRAINAGE}

ERCP is the standard of treatment for most biliary disorders; however, in patients with SAA, ERCP is associated with a low success rate and high complication rate. Reported rates of technical failure range from $13 \%$ to $67 \%$, and rates of perforation and mortality are approximately $18 \%$ and $3 \%$, respectively. Percutaneous transhepatic biliary drainage (PTBD) is a reasonable alternative; however, it can lead to external bile acid loss, as well as significant long-term costs, and burden to patients and their families. In addition, PTBD is associated with $0.5 \%-15 \%$ morbidity and $0-4.9 \%$ mortality. Therefore, EUS-BD has surfaced as a feasible alternative when ERCP fails. The average success rate of EUS-BD varies from $77 \%$ to $94 \%$ with a complication rate of $19 \%-27 \%$, which is comparable to, if not better than, PTBD. In addition, it is associated with lower long-term costs and fewer adverse events.

EUS-BD in patients with SAA can be classified using the approach taken to achieve access to the biliary system as well as the direction of the subsequent intervention. The two approaches used to access the biliary system are transmural, through the stomach or bowel wall, or transpapillary. Subsequent biliary intervention can then be performed using an antegrade or retrograde approach. Transmural access with a retrograde approach is commonly referred to as a rendezvous procedure. Procedures using transmural access with an antegrade approach fall under the category of antegrade biliary drainage. More recent approaches, such as external and internal EUS-directed transgastric ERCP (external EDGE and internal EDGE) procedures, have focused more on creating novel techniques to gain transpapillary access with subsequent retrograde interventions. Based on a review of current data, the overall success rate of EUS-guided biliary drainage, regardless of approach or underlying pathology, approaches $84 \%-93 \%{ }^{[4]}$ Here, we will provide a review of the various techniques and outcomes of EUS-guided biliary interventions in patients with SAA.

\section{ENDOSCOPIC ULTRASOUND-GUIDED RENDEZVOUS PROCEDURE}

In 1996, Wiersema et al. reported using EUS-guided access to the biliary tree after failed endoscopic retrograde cholangiography (ERC). Since then, multiple studies have shown that EUS-rendezvous (EUS-RV) is a feasible salvage technique to obtain access to the biliary system after failed ERC.

The rendezvous technique is considered as a bridging procedure in patients with endoscopically accessible papilla or biliary anastomotic site after failed ERCP cannulation. EUS-RV can be divided into an intrahepatic biliary duct (IHBD) approach and an extrahepatic biliary duct (EHBD) approach. These techniques achieve biliary access under EUS and fluoroscopic guidance by creating a temporary biliaryenteric fistula. ${ }^{[9]}$ A fine needle aspiration (FNA) needle is used to access the biliary system via the stomach or duodenum. Once accurate placement is achieved, the guidewire is advanced through the biliary duct 
and ampulla into the duodenum [Figure 1]. ERCP is reattempted, and biliary cannulation is achieved with the aid of EUS-placed guidewire [Figure 2].

The overall success rate of EUS-RV is $81 \%$ with a complication rate of $10 \% .{ }^{[9]}$ This is comparable for both IHBD and EHBD approaches. One of the most challenging aspects of EUS-RV is guidewire manipulation. The guidewire has to pass through the long rigid needle, biliary ducts, obstruction, and ampulla. These challenges are more pronounced with an IHBD, approach due to the longer distance between the needle tip and target which decreases the "pushability" of the advancing guidewire. Another challenge endoscopists face using IHBD approach is successfully puncturing the biliary ducts, which may be particularly difficult due to small duct size. Reported failure rate of IHBD approach by Maranki et al. is around 13\% (five of forty patients). The EHBD approach is from the duodenum, which decreases the distance between the biliary access point and target, thus allowing for better control of the guidewire. The biliary duct with EHBD approach is also larger; however, the trajectory tends to favor the liver hilum rather than the distal bile duct. Ideally, first approach should be EHBD from the second portion of the duodenum followed by the bulb, with an IHBD approach reserved for last.

EUS-RV technique is not without complications. One of the most feared complications in EUS-guided RV is bile leakage from the punctured biliary duct resulting in peritonitis. Other complications include pancreatitis, pneumoperitoneum, sepsis, and abdominal pain. The combined incidence of these five complications is around $13 \%$. The EHBD approach is associated with a greater risk of bile leak than with the IHBD approach.

\section{ENDOSCOPIC ULTRASOUND-GUIDED ANTEGRADE BILIARY DRAINAGE}

EUS-antegrade (EUS-AG) is considered in patients who failed rendezvous ERCP, are poor surgical candidates, or when rendezvous ERCP is not feasible due to altered surgical anatomy or outlet obstruction. EUSAG is similar to pancreatic pseudocyst drainage. In EUS-AG, access to the biliary system is achieved via an enterobiliary fistula and bypassing cannulation of the ampulla, as illustrated in Figure 3. Color Doppler is used to identify any interposing vessels, and IHBD is punctured from the intestine using an FNA needle followed by bile aspiration and cholangiography.

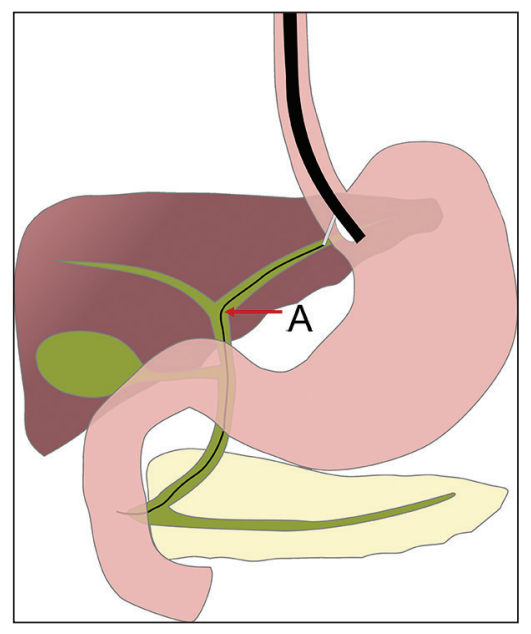

Figure 1. A guidewire is present in the biliary duct (A) and passing through the ampulla into the duodenum after endoscopic ultrasoundguided rendezvous procedure

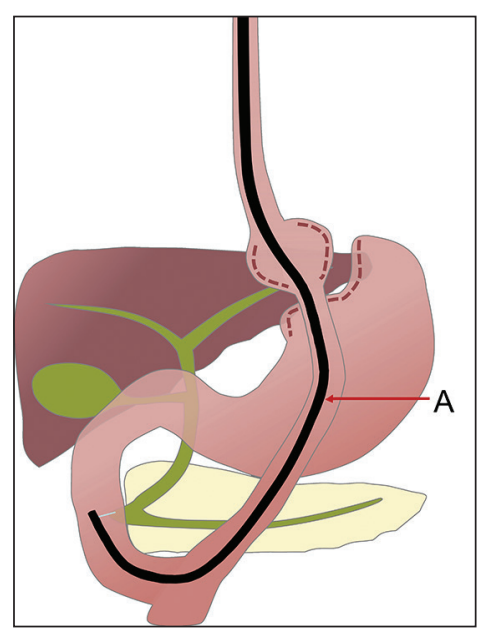

Figure 2. Endoscopic retrograde cholangiopancreatography with biliary cannulation using an enteroscope $(\mathrm{A})$ is achieved with the aid of the endoscopic ultrasound placed guidewire via the rendezvous technique in a patient with a Roux-en-Y gastric bypass

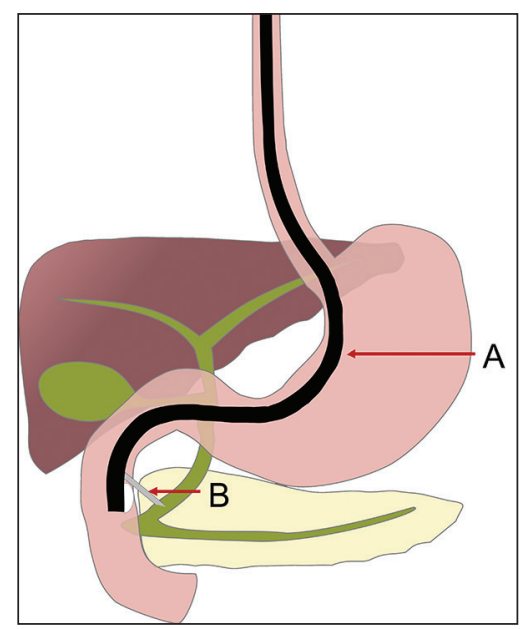

Figure 3. Endoscopic ultrasound-guided antegrade biliary drainage is performed using a linear echoendoscope (A). Under endoscopic ultrasound guidance, the common bile duct is punctured using a needle (B) 
Martin, et al.: Therapeutic EUS in altered anatomy

A guidewire is inserted, and a bougie dilator is used to dilate the fistula. The guidewire is advanced through the ampulla or anastomosis with coordinated movements of the guidewire and dilator inside the biliary system. Finally, biliary obstruction is relieved via self-expandable metallic stent or balloon dilation in an antegrade fashion. All devices are removed following successful biliary decompression.

Only several reports exist regarding one-step EUS-AG. The overall success is $77 \%$ with a complication rate of $5 \% \cdot{ }^{[7-9]}$ EUS-AG procedure is completed with an EUS scope alone and does not rely on deep insertion for access to the biliary orifice. Once the fistula is dilated with a bougie dilator, the guidewire can be easily advanced through bile ducts and the ampulla or anastomosis into the intestine with coordinated movements of the bougie dilator and the guidewire inside the biliary system. This is unlike EUS-RV where only the guidewire enters the biliary system. A major concern with EUS-AG is bile leak into the peritoneal cavity through the temporary fistula; however, no cases of biliary peritonitis have been reported. ${ }^{[10]}$ EUS-AG is considered as a first-line intervention for patients with an inaccessible ampulla after failed ERCP, with the exception of patients with duodenal invasion. Shortterm outcome of EUS-AG for SAA patients has been promising though no long-term data are available.

\section{ENDOSCOPIC ULTRASOUND- DIRECTED TRANSGASTRIC ENDOSCOPIC RETROGRADE CHOLANGIOPANCREATOGRAPHY (EUS-DIRECTED TRANSGASTRIC ERCP)}

In patients who have undergone Roux-en-Y reconstruction, successful intubation of the afferent limb in the $\mathrm{Y}$ anastomosis can be particularly challenging. The long limb that transverses from gastrojejunal orifice to jejunojejunal anastomosis to reach the afferent small-bowel limb and the long limb is often $>100 \mathrm{~cm}$. In addition, the angulation of the jejunojejunal anastomosis can be up to $180^{\circ}$ and adhesions from surgery add to the challenge.

ERCP, with a side-viewing endoscope, in Roux-en-Y gastric bypass (RYGB) patients, was previously not considered possible until 1998 when Baron and Vickers first described this technique using surgical gastrostomy. Since then, there have been modifications to these methods, most notably the external EDGE procedure and the internal EDGE procedure. ${ }^{[10]}$

\section{EXTERNAL EUS-DIRECTED TRANSGASTRIC ERCP PROCEDURE}

The EDGE procedure was first described in the literature by Kedia et al., in 2014. ${ }^{[10]}$ The procedure is used for pancreaticobiliary drainage in patients who have undergone a prior RYGB surgery. The procedure is done in two stages and involves the placement of an EUS-assisted percutaneous endoscopic gastrostomy (PEG) tube with subsequent antegrade ERCP through the created gastrostomy tract. EUS-assisted PEG tube placement was first described by Attam et al. as a novel way to access the gastric remnant in patients with prior RYGB ${ }^{[8]}$ Kedia et al. adopted this technique to complete the first stage of the EDGE procedure.

In the first stage, a linear echoendoscope is used to endosonographically identify the gastric remnant from the gastric pouch. The gastric remnant is then accessed with a needle under EUS guidance through which saline or air is injected into the remnant stomach to confirm positioning. A guidewire is then passed through the EUS needle and coiled in the gastric remnant. A 16 Fr PEG tube is subsequently placed under fluoroscopic guidance into the gastric remnant.

The second stage of the procedure is performed between 3 and 9 days after the first stage. The second stage involves exchanging the already placed PEG tube with a transcutaneous fully covered metal stent. An antegrade ERCP is then performed through the gastrocutaneous stent allowing for any necessary pancreaticobiliary drainage. No large-scale clinical trials have been conducted to assess the success and complication rates of the EDGE procedure. The largest case series in the literature includes 6 patients with RYGB anatomy who underwent EDGE procedure. Five cases were for cholelithiasis and 1 for biliary stricture. EUS-guided access into the excluded stomach remnant was successful in all 6 patients $(100 \%)$. Antegrade ERCP was also successfully performed in all 6 patients $(100 \%)$. Two patients developed a localized infection at the site of PEG insertion requiring a course of oral antibiotics. There were no adverse events associated with the antegrade ERCP portion of the procedure.

The EDGE procedure is a minimally invasive approach to obtain pancreaticobiliary access in patients with prior 


\section{Martin, et al:: Therapeutic EUS in altered anatomy}

RYGB. The major advantages of the procedure include: A single team can perform the entire procedure, it is minimally invasive, and the success rate based on current data has the potential to exceed that of the other current treatment modalities. The data regarding the efficacy of the EDGE procedure are currently limited; however, Kedia et al's small case series showed a $100 \%$ success rate of accessing the gastric remnant and $100 \%$ success in performing subsequent antegrade ERCP. The major downsides of the EDGE procedure are that it is performed in two stages and requires a transcutaneous approach. This technique would not be an option for patients who require immediate intervention given that the procedure takes a median of 5.8 days to complete. The procedure also requires the formation of a transcutaneous gastrostomy tract, which can cause localized infections, scarring, and fistula formation. Given that it is a new procedure, the longterm adverse effects of the EDGE procedure have not yet been realized.

\section{INTERNAL EUS-DIRECTED TRANSGASTRIC ERCP PROCEDURE}

As opposed to the transcutaneous approach of the external EDGE procedure, the internal EDGE procedure uses a lumen-opposing metal stent to access the gastric remnant and perform an antegrade ERCP in a single endoscopy session. Kedia et al. first introduced the procedure in 2014 shortly after their case series describing the external EDGE procedure. Unlike the external EDGE procedure, the internal EDGE procedure allows for pancreaticobiliary intervention in a single session. The first step of the procedure is to identify the excluded gastric remnant using a linear echoendoscope from the gastric pouch. Once the gastric remnant is identified, a 19-guage EUS needle is passed from the gastric pouch into the gastric remnant. Contrast and saline are injected into the gastric remnant to confirm positioning. A guidewire is passed into the gastric remnant and using a $4 \mathrm{~mm}$ balloon, the tract is dilated. Following balloon dilation of the tract, a fully covered lumen-opposing metal stent (AXIOS stent) is placed across the tract creating a gastrogastric fistula between gastric pouch and excluded gastric remnant. The lumen of the stent is dilated to $18 \mathrm{~mm}$ using a dilating balloon, which creates a conduit through which a duodenoscope can be passed and an antegrade ERCP can be performed. A second endoscopy is completed weeks after the initial procedure to remove the lumen-opposing metal stent and repair the remnant gastric pouch fistula. ${ }^{[10,11]}$
This novel procedure addresses many of the issues associated with the external EDGE procedure. The internal EDGE procedure is completed in a single session and can achieve urgent pancreaticobiliary drainage in patients who require immediate intervention. The internal EDGE procedure is also less invasive. It eliminates the need to create a transcutaneous gastrostomy tract, which will reduce cutaneous infection rates, and eliminate scarring and cutaneous fistula formation. There is only one case report in the current literature describing the use of the internal EDGE procedure and, thus, the data on the effectiveness and complications associated with the internal EDGE procedure are very limited. Some of the potential complications of the Internal EDGE procedure include stent migration during ERCP, stent migration following the procedure, postprocedural weight gain with a maintained gastrogastric fistula, and permanent fistula formation. More literature is needed to determine the short- and long-term complications, complication rate, and success rate of the internal EDGE procedure.

\section{ENDOSCOPIC ULTRASOUND-GUIDED PANCREATIC DRAINAGE}

EUS-pancreatic drainage (EUS-PD) has evolved as an attractive alternative to surgery and ERCP in patients with SAA. EUS-PD was first described by Harada et al. in 1995 and has drastically evolved since then. ${ }^{[12]}$ Common indications for EUS-PD in patients with SAA include stenosis of the pancreaticoenterostomy after pancreaticoduodenectomy (observed in approximately $30 \%$ of these patients), ${ }^{[13]}$ pancreatic fistula along with stenotic pancreaticojejunal (PJ) anastomosis (seen in $10 \%-20 \%$ of Whipple patients), ${ }^{[14-19]}$ acute recurrent pancreatitis (seen in 2\%-3\% of Whipple patients), ${ }^{[18,19]}$ chronic pancreatitis, pancreatic duct strictures, pancreatic duct stones, and failed ERCP.

Before conducting EUS-PD, both indications and contraindications of the procedure should be considered. Relative contraindications include thrombocytopenia (platelet count $<50,000$ ), coagulopathy (international normalized ratio $>1.5$ ), and hemodynamic instability. Magnetic resonance cholangiopancreatography has been routinely performed in many of the case studies and reviews to assist in characterization of the patient's anatomy. Prophylactic intravenous or oral antibiotics are given before the procedure in case of leakage of gastric, biliary, pancreatic, or enteric contents and occasionally 
Martin, et al.: Therapeutic EUS in altered anatomy

continued for up to 7 days postprocedure. ${ }^{[20-23]}$ Curved linear array echoendoscopes are routinely used. FNA needles utilized include 19G, 22G, and 25G. Guidewires range from $0.018^{[24,25]}$ to 0.035 inch $^{[4]}$ but will vary depending on the gauge of the FNA needle used. Dilation of the needle tract (using dilation catheters and/or balloon catheters) is mandatory before stenting and depends on the size of the pancreatic duct stent. Plastic stents (5 Fr or 7 Fr) typically used as fully or partially covered self-expendable metallic stents can block the pancreatic duct side branches potentially resulting in pancreatitis. It is vital to identify the optimal route by which the main pancreatic duct (MPD) will be accessed. Ideal scenarios include the shortest distance between EUS transducer and MPD, lack of structural obstacles (including vasculature), and optimal angles and pathways for instrumentation and eventual stent placement. ${ }^{[19]}$ Once MPD is visualized under EUS guidance, the appropriate FNA needle is used to transgastrically puncture MPD. Although the more common route is transgastrically (endoscopic pancreaticogastrostomy), a transbulbar approach has also been used (endoscopic pancreaticobulbostomy). ${ }^{[23]}$ Fluoroscopic verification of MPD access is performed with contrast injection. A guidewire is then passed through the FNA needle into MPD under fluoroscopic guidance.

At this point, PD can be achieved using two different techniques such as transmural pancreatic duct stenting (antegrade) or retrograde stenting (rendezvous). ${ }^{[25,26]}$ With transmural pancreatic duct stenting, the entire procedure is performed using the echoendoscope with the stent having to traverse MPD into the stomach. The transmural pancreatic duct stenting technique, therefore, requires significant dilation which can result in adverse events such as pancreatic juice leakage, pancreatitis, bleeding, and perforation. ${ }^{[25,26]}$ In the retrograde (rendezvous) approach, MPD is similarly accessed transgastrically (rarely transduodenally or transjejunally) under EUS guidance. ${ }^{[4]}$ The guidewire is advanced through the papilla and into the duodenum, making sure that there is sufficient looping within the duodenum to facilitate the next step. The echoendoscope is then removed; however, the guidewire remains in place. Subsequently, either a side-viewing duodenoscope, colonoscope, or balloon enteroscope can be passed through the gastrojejunostomy to the papilla and site of anastomosis. ${ }^{[4,24,25]}$ Biopsy forceps are used to grasp the guidewire, and the papilla can be dilated accordingly before stent placement. If during the initial steps, the guidewire cannot be passed through the entire MPD due to structural obstacles (e.g., tight stricture) or other technical issues (angle of EUS and needle puncture), the rendezvous method cannot be completed.

A newer technique used to facilitate EUS-PD is the pancreatic antegrade needle-knife whereby a cut is made at the PJ anastomosis site, creating a pancreaticojejunostomy. ${ }^{[23]}$ The pancreaticojejunostomy is dilated and a long plastic stent (up to $15 \mathrm{~cm}$ ) is placed extending from the jejunum to the stomach. ${ }^{[23]}$ Although data are relatively limited, the technical success rates in patients with both normal and SAA have been reported as between $25 \%$ and $100 \%$ for EUS-guided transmural pancreatic duct stenting and $77 \%-92 \%$ for the EUSguided rendezvous technique. ${ }^{[26-28]}$ Complications in all patients have been reported between $10 \%$ and $43 \%{ }^{[19,29]}$ A review conducted by Prichard et al. ${ }^{[26]}$ identified patients with SAA who underwent EUSguided pancreatic duct drainage via either technique. Many of the studies reviewed were small and included patients with both normal anatomy and SAA. The SAA cohorts were especially limited, with most studies containing $<15$ SAA patients who underwent any form of EUS-guided PD. With respect to SAA, there were considerably more data in post-Whipple patients compared to those that are postgastric bypass. Many of the studies did not specifically detail which patients (normal anatomy vs. SAA) had technical and clinical success, complications, and exact length of followup; therefore, exact calculation of these rates was challenging.

Upon further review of the literature, there were approximately eight studies that had at least seven patients with SAA. ${ }^{[21-28]}$ As many of the cohorts contained limited number of patients with varying medical history, much of the data reported were heterogeneous. The 96 patients identified underwent EUS-guided antegrade pancreatic duct stenting and rendezvous technique at similar rates. The definition of "technical success" and identification of which patients with SAA achieved is varied between studies, but rates ranged from $44 \%$ to $100 \%$. Similarly, "clinical success" had a much more variable definition between studies and ranged between $22 \%$ and 100\%. The most common "mild" complications reported in patients undergoing EUS-guided PD included abdominal pain, minor bleeding (controlled with hemoclip), and pancreatitis with rates between $5.8 \%$ and $29 \%$. Serious complications (e.g., perforation, peripancreatic 
Martin, et al:: Therapeutic EUS in altered anatomy

abscesses, and pancreatic juice leakage) ranged from $0 \%$ to $12.5 \%$. Median follow-up after the procedure was between 8 and 47 months (3-108 months). An interesting postprocedural issue that was reported in several studies included stent dysfunction and the requirement for additional procedures. As with the data mentioned above, many of the studies did not specify stent dysfunction rates specifically in patients with SAA; however, rates have been reported as between $25 \%$ and $55 \%$. ${ }^{[22,25,29]}$

Technical and clinical success was impacted by multiple factors. Many of the studies reported difficulties with MPD access when it was not dilated. ${ }^{[4,28]}$ Suboptimal angles during initial attempts at access and tight strictures within MPD can often lead to passage of the guidewire into the pancreatic duct side branches. ${ }^{[4,26,28]}$ Many of the studies reported the need for repeated attempts at EUS-PD, which could be considered burdensome to both patient and endoscopist. However, additional EUS-PD attempts may prove beneficial to patients as Kikuyama et al. reported increased technical success rates in this scenario. ${ }^{[22]}$

The utilization of EUS-PD in patients with SAA is an evolving technique that is continuing to accumulate data. Although EUS-PD in patients with SAA requires a high level of skill and experience, it offers a potentially useful alternative to surgery or percutaneous intervention in the patients requiring pancreatic duct decompression. Additional studies in patients with SAA are needed to further determine the exact role of EUS-PD and characterize both efficacy and complication rates especially with respect to long-term outcomes.

\section{CONCLUSION}

EUS-BD and EUS-PD are reasonable salvage techniques for patients requiring pancreaticobiliary intervention in whom traditional ERCP is not possible because of SAA or the inability to access the papillae secondary to obstruction. More studies are required to further compare the efficacy and safety of these procedures compared to percutaneous, laparoscopic, surgical, or OAE techniques. Advanced endoscopists must also become more experienced with these techniques before becoming more widely disseminated.

\section{Financial support and sponsorship}

Nil.
Conflicts of interest

There are no conflicts of interest.

\section{REFERENCES}

1. Nguyen NT, Masoomi H, Magno CP, et al. Trends in use of bariatric surgery, 2003-2008. J Am Coll Surg 2011;213:261-6.

2. Siegel RL, Miller KD, Jemal A. Cancer statistics, 2015. CA Cancer J Clin 2015;65:5-29.

3. Skinner M, Popa D, Neumann H, et al. ERCP with the overtube-assisted enteroscopy technique: A systematic review. Endoscopy 2014;46:560-72.

4. Gutierrez JM, Lederer H, Krook JC, et al. Surgical gastrostomy for pancreatobiliary and duodenal access following Roux en $\mathrm{Y}$ gastric bypass. J Gastrointest Surg 2009;13:2170-5.

5. Nosher JL, Bodner LJ, Girgis WS, et al. Percutaneous gastrostomy for treating dilatation of the bypassed stomach after bariatric surgery for morbid obesity. AJR Am J Roentgenol 2004;183:1431-5.

6. Stein EG, Cynamon J, Katzman MJ, et al. Percutaneous gastrostomy of the excluded gastric segment after Roux-en-Y gastric bypass surgery. J Vasc Interv Radiol 2007;18:914-9.

7. Kahaleh M, Artifon EL, Perez-Miranda M, et al. Endoscopic ultrasonography guided drainage: Summary of consortium meeting, May 21, 2012, San Diego, California. World J Gastroenterol 2015;21:726-41.

8. Attam R, Leslie D, Freeman M, et al. EUS-assisted, fluoroscopically guided gastrostomy tube placement in patients with Roux-en-Y gastric bypass: A novel technique for access to the gastric remnant. Gastrointest Endosc 2011;74:677-82.

9. Kedia P, Kumta NA, Sharaiha R, et al. Bypassing the bypass: EUSdirected transgastric ERCP for Roux-en-Y anatomy. Gastrointest Endosc 2015;81:223-4.

10. Kedia P, Sharaiha RZ, Kumta NA, et al. Internal EUS-directed transgastric ERCP (EDGE): Game over. Gastroenterology 2014;147:566-8.

11. Iqbal S, Friedel DM, Grendell JH, et al. Outcomes of endoscopicultrasound-guided cholangiopancreatography: A literature review. Gastroenterol Res Pract 2013;2013:869214.

12. Harada N, Kouzu T, Arima M, et al. Endoscopic ultrasound-guided pancreatography: a case report. Endoscopy 1995;27:612-5.

13. Fujii-Lau LL, Levy MJ. Endoscopic ultrasound-guided pancreatic duct drainage. J Hepatobiliary Pancreat Sci 2015;22:51-7.

14. Takano S, Ito $\mathrm{Y}$, Oishi $\mathrm{H}$, et al. A retrospective analysis of 88 patients with pancreaticogastrostomy after pancreaticoduodenectomy. Hepatogastroenterology 2000;47:1454-7.

15. Schlitt HJ, Schmidt U, Simunec D, et al. Morbidity and mortality associated with pancreatogastrostomy and pancreatojejunostomy following partial pancreatoduodenectomy. Br J Surg 2002;89:1245-51.

16. McKay A, Mackenzie S, Sutherland FR, et al. Meta-analysis of pancreaticojejunostomy versus pancreaticogastrostomy reconstruction after pancreaticoduodenectomy. Br J Surg 2006;93:929-36.

17. Haddad LB, Scatton $O$, Randone B, et al. Pancreatic fistula after pancreaticoduodenectomy: The conservative treatment of choice. НРB (Oxford) 2009;11:203-9.

18. Chahal P, Baron TH, Topazian MD, et al. Endoscopic retrograde cholangiopancreatography in post-Whipple patients. Endoscopy 2006;38:1241-5.

19. Kinney TP, Li R, Gupta K, et al. Therapeutic pancreatic endoscopy after Whipple resection requires rendezvous access. Endoscopy 2009;41:898-901.

20. Farrell J, Carr-Locke D, Garrido T, et al. Endoscopic retrograde cholangiopancreatography after pancreaticoduodenectomy for benign and malignant disease: Indications and technical outcomes. Endoscopy 2006;38:1246-9.

21. Tessier G, Bories E, Arvanitakis M, et al. EUS-guided pancreatogastrostomy and pancreatobulbostomy for the treatment of pain in patients with pancreatic ductal dilatation inaccessible for transpapillary endoscopic therapy. Gastrointest Endosc 2007;65:233-41. 


\section{Martin, et al:: Therapeutic EUS in altered anatomy}

22. Kikuyama M, Itoi $\mathrm{T}$, Ota $\mathrm{Y}$, et al. Therapeutic endoscopy for stenotic pancreatodigestive tract anastomosis after pancreatoduodenectomy (with videos). Gastrointest Endosc 2011;73:376-82.

23. Barkay O, Sherman S, McHenry L, et al. Therapeutic EUS-assisted endoscopic retrograde pancreatography after failed pancreatic duct cannulation at ERCP. Gastrointest Endosc 2010;71:1166-73.

24. Wilson JA, Hoffman B, Hawes RH, et al. EUS in patients with surgically altered upper GI anatomy. Gastrointest Endosc 2010;72:947-53.

25. Moreels TG. Endoscopic retrograde cholangiopancreatography in patients with altered anatomy: How to deal with the challenges? World J Gastrointest Endosc 2014;6:345-51.
26. Prichard D, Byrne MF. Endoscopic ultrasound guided biliary and pancreatic duct interventions. World J Gastrointest Endosc 2014;6:513-24.

27. Iwashita T, Lee JG. Endoscopic ultrasonography-guided biliary drainage: Rendezvous technique. Gastrointest Endosc Clin N Am 2012;22:249-58, viii-ix.

28. Ryou M, Mullady DK, Dimaio CJ, et al. Pancreatic antegrade needle-knife (PANK) for treatment of symptomatic pancreatic duct obstruction in Whipple patients (with video). Gastrointest Endosc 2010;72:1081-8.

29. Ergun M, Aouattah T, Gillain C, et al. Endoscopic ultrasound-guided transluminal drainage of pancreatic duct obstruction: long-term outcome. Endoscopy 2011;43:518-25. 